\title{
Physics and Dynamics of Density-Compensated Temperature and Salinity Anomalies. Part I: Theory
}

\author{
RÉmi TAILleuX \\ IPSL-Laboratoire de Météorologie Dynamique, UPMC, Paris, France, and EGS-CSAG, University of Cape Town, Cape Town, \\ South Africa
}

Alban LAZAR

Laboratoire d'Océanographie Dynamique et de Climatologie, Paris, France

C. J. C. REASON

Oceanography Department, University of Cape Town, Cape Town, South Africa

(Manuscript received 1 December 2003, in final form 17 September 2004)

\begin{abstract}
Subducted temperature anomalies have been invoked as a possible way for midlatitudes to alter the climate variability of equatorial regions through the so-called thermocline bridge, both in the Pacific and Atlantic Oceans. To have a significant impact on the equatorial heat balance, however, temperature anomalies must reach the equatorial regions sufficiently undamped. In the oceans, the amplitude of propagating temperature (and salinity) anomalies can be altered both by diabatic (nonconservative) and adiabatic (conservative) effects. The importance of adiabatic alterations depends on whether the anomalies are controlled by wave dynamics or by passive advection associated with density compensation. Waves being relatively well understood, this paper seeks to understand the amplitude variations of density-compensated temperature and salinity anomalies caused by adiabatic effects, for which no general methodology is available. The main assumption is that these can be computed independent of amplitude variations caused by diabatic effects. Because density compensation requires the equality $T^{\prime} / S^{\prime}=\beta_{S} / \alpha$ to hold along mean trajectories, the ratio $T^{\prime} / S^{\prime}$ may potentially undergo large amplitude variations if the ratio $\beta_{S} / \alpha$ does, where $\alpha$ and $\beta_{S}$ are the thermal expansion and haline contraction coefficients, respectively. In the oceans, the ratio $\beta_{S} / \alpha$ may decrease by an order-1 factor between the extratropical and tropical latitudes, but such large variations are in general associated with diapycnal rather than isopycnal motion and hence are likely to be superimposed in practice with diabatically induced variations. To understand the individual variations of $T^{\prime}$ and $S^{\prime}$ along the mean streamlines, two distinct theories are constructed that respectively use density/salinity and density/spiciness as prognostic variables. If the coupling between the prognostic variables is neglected, as is usually done, both theories predict at leading order that temperature (salinity) anomalies should be systematically and significantly attenuated (conserved or amplified), on average, when propagating from extratropical to tropical latitudes. Along particular trajectories following isopycnals, however, both attenuation and amplification appear to be locally possible. Assuming that the density/spiciness formulation is the most accurate, which is supported by a theoretical assessment of higher-order effects, the present results provide an amplification mechanism for subducted salinity anomalies propagating equatorward, by which the latter could potentially affect decadal equatorial climate variability through their slow modulation of the equatorial mixed layer, perhaps more easily than their attenuated temperature counterparts. This could be by affecting, for instance, barrier layers by which salinity is known to strongly affect local heat fluxes and heat content.
\end{abstract}

\section{Introduction}

A current central issue in ocean climate theory is to understand the possible links between the variability of

Corresponding author address: Dr. Rémi Tailleux, EGS-CSAG, University of Cape Town, Private Bag, Rondebosch 7701, South Africa.

E-mail: tailleux@lmd.jussieu.fr temperature and salinity anomalies and that of weather and climate fluctuations on the intraseasonal to decadal time scales. In the current state of our knowledge, salinity anomalies are known to be climatically important on long time scales because they affect the variability of the thermohaline circulation (Rahmstorf 1995; Mikolajewicz and Maier-Reimer 1990) as well as the rate of deep-water formation. Regarding temperature anomalies, studies linking sea surface temperature (SST) to 
weather and climate patterns in various parts of the world are numerous and ever expanding, even though the precise mechanisms by which such control is achieved is yet to be completely understood. As a result, SST prediction has become central to current seasonal forecasting strategies. For all these reasons, it is of primary importance to understand the dynamical and thermodynamical processes accounting for the creation, evolution, and eventual demise of temperature and salinity anomalies in the oceans.

From observations, there is evidence of two main kind of large-scale propagating disturbances affecting oceanic and potentially climate variability, namely westward propagating anomalies, associated with firstmode baroclinic Rossby waves, and second, advected or seemingly advected anomalies. The existence of Rossby waves is ubiquitous in satellite altimeter data (Chelton and Schlax 1996; Polito and Cornillon 1997), in infrared data (Hill et al. 2000), possibly even the sea surface color data (Cipollini et al. 2001; Quartly et al. 2003) and hydrographic data (see Fu and Chelton 2001 for a review). First-mode baroclinic Rossby waves play a central role in the adjustment response to change in the wind forcing (Sirven and Frankignoul 2000) and explain the main part of wind-forced decadal variability (Sturges and Hong 1995). These waves might be possibly involved in coupled ocean-atmosphere decadal modes of variability (Gallego and Cessi 2000; White et al. 1998). Advected or seemingly advected anomalies have been observed in the Pacific Ocean thermocline (Deser et al. 1996; Zhang and Levitus 1997) as well as in the surface (Sutton and Allen 1997), mainly in the temperature field. In the same category, but mostly visible in the salinity field is the great salinity anomaly (Dickson et al. 1988). Seemingly advected temperature anomalies have been hypothesized to affect the climate variability of the equatorial regions through the "thermocline bridge" in the Pacific by Gu and Philander (1997) as well as in the Atlantic Ocean by Lazar et al. (2001).

It is usually assumed (e.g., Schneider 2000) that large-scale propagating temperature and salinity anomalies fall broadly into two main dynamically distinct categories: 1) wavelike anomalies having a density signature and a propagation path that may depart significantly from that of the mean streamlines; and 2) density-compensated (spiciness) anomalies having no density signature, and hence simply passively advected by the mean circulation. Since the mean circulation is not purely westward in general, there is no doubt that westward propagating signals belong to the wave category, the theory of which has progressed rapidly in the past few years (Killworth et al. 1997; Dewar 1998; Tailleux and McWilliams 2001; Killworth and Blundell 2003a). In contrast, the nature of the observed seemingly advected anomalies is still under debate, with proponents of a wave explanation in terms of a second baroclinic mode (Liu 1999a,b; Liu and Shin 1999; Huang and Pedlosky 1999; Sirven and Frankignoul
2000; Killworth and Blundell 2001), as well as of in terms of density-compensated anomalies (Lazar et al. 2001; Schneider 2000, 2004; Lukas 2001).

Except in a few cases (e.g., Lukas 2001), it is usually not possible for lack of adequate salinity data to directly determine whether seemingly advected anomalies should be explained in terms of wave dynamics or as passively advected density-compensated anomalies. To make progress, it would be helpful to have more theoretical knowledge about the distinctive features of each kind of anomalies. So far, however, the dynamics of the second baroclinic mode has essentially been studied by means of layered models (Liu 1999a,b) or a truncated normal mode approach (Killworth and Blundell 2001), whereas the study of density-compensated anomalies has relied exclusively on the use of numerical general circulation models (Lazar et al. 2001; Zhang et al. 2001; Schneider 2004). As a result, theoretical knowledge for these two kinds of anomalies appears to be severely lacking for a realistic continuously stratified fluid. In fact, we are not even aware of any mathematical derivation justifying the existence of densitycompensated anomalies from first principles! For these reasons, it seems important and timely to investigate the fundamental properties of density-compensated temperature and salinity anomalies, to lay down the theoretical foundations for their study and understanding.

Several questions arise. First, what is the mathematical theory behind density-compensated anomalies, as opposed to that for waves? Are density-compensated anomalies an exact solution of the equations of motion, or an approximate one? If so, what are the underlying approximations? Is it density or potential density that is implied in the term "density" compensated? What does it exactly mean when we say that density-compensated temperature and salinity anomalies are passively advected? Does that necessarily imply that such anomalies are conserved along the mean streamlines? In this paper, we show that density-compensated anomalies are only an approximation to the equations of motion, in the sense that actual such anomalies should always possess a signature, if only a small one, in the pressure and density fields, but which it is justified to neglect at leading order. Furthermore, it is also shown that the passively advective character of density-compensated temperature/salinity $(T / S)$ anomalies does not imply that the latter are conserved along the mean streamlines. In other words, density-compensated $T / S$ anomalies may undergo significant amplitude variations. In this respect, the present paper represents a significant advance for the general theoretical understanding of amplitude variations of $T / S$ anomalies in the ocean, which complements that already achieved for waves. In the latter case, indeed, it is possible to derive amplitude equations for wave motions from so-called wave action conservation laws, which often amounts to a statement about energy conservation. In that case, the basic physi- 
cal principle is that the wave amplitude increases as a result of ray focusing, and decrease as a result of ray widening. No comparable simple physical principle has been derived yet for density-compensated $T / S$ anomalies. Probably, this is because it was not realized before that such anomalies could undergo amplitude variations in absence of mixing, which is one of the main results of this paper.

The dynamics of density-compensated $T / S$ anomalies is closely tied to the extensively studied process of subduction, that is, the process by which anomalies are transferred from the mixed layer to the main thermocline. Physically, subducted density-compensated $T / S$ anomalies are created in the mixed layer as the result of coupled air-sea interactions whose details will ultimately decide of their amplitude, the theory for which is based on Stommel's Ekman layer "demon" (e.g., Stommel 1979; Williams et al. 1995). In the past, subduction has mainly been studied within the context of the so-called ventilated thermocline theory (e.g., Luyten et al. 1983), and the emphasis put on the determination of the main trajectories followed by the anomalies once in the thermocline, not on their amplitude variations. In particular, the main quantity emphasized in subduction studies is potential vorticity, for the latter is believed to be a materially conserved quantity, and hence a marker of the main trajectories followed by subducted parcels. In the present paper, on the other hand, we assume the main trajectories to be known, in order to focus on the amplitude variations of $T / S$ anomalies along them. Such an issue cannot be easily addressed in most current theoretical frameworks, which usually do not separate the contribution of temperature and salinity on density.

After subduction in the ocean interior, densitycompensated $T / S$ anomalies undergo amplitude changes caused both by diabatic and adiabatic processes. Among the diabatic processes bound to alter the amplitude of density-compensated anomalies, one may invoke turbulent mixing, instabilities, and the breaking of internal waves, all of which have been and still are the focus of numerous studies seeking to quantify their effects, in order to help to the design of subgridscale parameterization scheme in general circulation models for instance. On the other hand, we are not aware of any real understanding of how adiabatic effects can impact on the amplitude of such anomalies, so the main purpose of this paper is to provide the first step toward such an understanding as predicted by the simplest linear theory, which allows us to identify and isolate one particular effect related to the spatial variations of the thermodynamic coefficients of seawater. The present paper should therefore not be regarded as a complete theory for the amplitude of actual density-compensated $T / S$ anomalies, both because it completely excludes important diabatic effects, as well as other potential adiabatic effects involving the coupling with waves via nonlinear and/or linear interactions. In the latter case, how- ever, a more sophisticated framework would be needed, which is hence deferred to a future study. Most likely, a full and complete study of the present issue requires the use of sophisticated eddy-resolving general circulation models; the present study should nevertheless be useful in providing some clues as to the way the solutions should be analyzed.

To proceed, we start with the full equations of motion formulated in terms of salinity and entropy, which are the natural conserved variables in absence of forcing and dissipation. Such equations are linearized and rewritten to obtain linearized prognostic equations for density and salinity anomalies in section 2. Section 3 discusses the general solution of the equations previously derived and shows how the spiciness mode is to be obtained at leading order. It also discusses the respective temperature and salinity signatures of the spiciness mode. The main result here is the prediction that the temperature signature of the spiciness mode is attenuated along mean streamlines when propagating equatorward, in contrast to the salinity signature which is conserved. Section 4 addresses the issues of robustness and accuracy of the previous result by showing that a different choice of prognostic variables, namely density and spiciness, yields significantly different leadingorder prediction. By computing the second-order effects of the density/salinity formulation, it is suggested that the density/spiciness formulation is the one yielding the most accurate leading-order prediction. The implication is that the temperature anomalies should be less attenuated than predicted by the density/salinity theory, whereas salinity anomalies should be amplified rather than strictly conserved. Section 5 offers a discussion of the results and of their potential consequences for climate.

\section{Model formulation and basic notations}

\section{a. Equations of motion}

We consider as our starting point the oceanic equations of motion formulated in terms of the threedimensional velocity field $\mathbf{v}=(u, v, w)$, the pressure $p$, in situ density $\rho$, entropy $\eta$, salinity $S$, and geopotential $\Phi=g z$, namely

$$
\begin{aligned}
\frac{D \mathbf{v}}{D t}+2 \boldsymbol{\Omega} \times \mathbf{v}+\frac{1}{\rho} \nabla p-\nabla \Phi & =\mathbf{F}_{\mathbf{v}}, \\
\frac{D \rho}{D t}+\rho \nabla \cdot \mathbf{v} & =0, \\
\frac{D \eta}{D t} & =\mathcal{F}_{\eta}, \\
\frac{D S}{D t} & =\mathcal{F}_{s}, \quad \text { and } \\
\rho & =\rho(\eta, S, p)
\end{aligned}
$$


(Salmon 1998), which express the conservation of momentum, mass, entropy, and salinity, respectively; here $\Omega$ is the earth's rotation vector, while $\mathbf{F}_{\mathbf{v}}, \mathcal{F}_{\eta}$, and $\mathcal{F}_{S}$ represent source and sink terms for the momentum, entropy, and salinity, respectively. We focus thereafter on the dynamics of anomalies, so that we write each variable as the sum of its mean and fluctuating parts. Retaining only the linear parts of the equations yields

$$
\begin{aligned}
\mathcal{L}\left(\mathbf{v}^{\prime}, \rho^{\prime}, p^{\prime}\right) & =0, \\
\frac{\partial \eta^{\prime}}{\partial t}+\overline{\mathbf{v}} \cdot \nabla \eta^{\prime}+\mathbf{v}^{\prime} \cdot \nabla \bar{\eta} & =0, \quad \text { and } \\
\frac{\partial S^{\prime}}{\partial t}+\overline{\mathbf{v}} \cdot \nabla S^{\prime}+\mathbf{v}^{\prime} \cdot \nabla \bar{S} & =0,
\end{aligned}
$$

where (6) is a formal way to write the linearization of (1)-(2) whose specific form is unimportant for the study of density-compensated anomalies (but which becomes crucial to study wave dynamics), and hence left unspecified. For simplicity, source terms for entropy and salinity were also discarded.

\section{b. Coupled salinity and density perturbations}

The above coupled equations constitute a system of coupled equations for seven variables $(u, v, w, \rho, p, S$, $\eta)$. This system can be simplified by using the equation of state (5) to reduce this number to six by eliminating entropy. To that end, the equation of state (5) is linearized around mean variables, which yields

$$
\rho^{\prime}=\rho_{S} S^{\prime}+\rho_{\eta} \eta^{\prime}+c_{s}^{-2} p^{\prime},
$$

where $\rho_{p}=1 / c_{s}^{2}$ is the inverse of the speed of sound squared, while expressions for $\rho_{S}$ and $\rho_{\eta}$ are provided in the appendix. To simplify notations, an overbar is omitted for the thermodynamic coefficients that are estimated for the mean values of $\eta, S$, and $p$. By multiplying (7) by $\rho_{\eta}$ and adding the result to (8) multiplied by $\rho_{S}$, we obtain after some manipulation:

$$
\frac{\partial \rho_{\theta}^{\prime}}{\partial t}+\overline{\mathbf{v}} \cdot \nabla \rho_{\theta}^{\prime}+\mathbf{v}^{\prime} \cdot \nabla \bar{\rho}_{\theta}=\varpi_{S} \rho_{S} S^{\prime}+\varpi_{\rho} \rho_{\theta}^{\prime}
$$

where we have introduced the following notations:

$$
\begin{aligned}
\rho_{\theta}^{\prime} & =\rho^{\prime}-\frac{1}{c_{s}^{2}} p^{\prime}, \\
\nabla \bar{\rho}_{\theta} & =\nabla \bar{\rho}-\frac{1}{c_{s}^{2}} \nabla \bar{P} \\
\varpi_{S} & =\frac{\rho_{\eta} \overline{\mathbf{v}} \cdot \nabla \rho_{S}-\rho_{S} \overline{\mathbf{v}} \cdot \nabla \rho_{\eta}}{\rho_{S} \rho_{\eta}}=\overline{\mathbf{v}} \cdot \nabla \ln \left|\frac{\rho_{S}}{\rho_{\eta}}\right|,
\end{aligned}
$$

and

$$
\varpi_{\rho}=\overline{\mathbf{v}} \cdot \nabla \ln \rho_{\eta}{ }^{1}
$$

\footnotetext{
${ }^{1}$ Note that (12) is a convenient abuse of notation since the curl of the right-hand side does not vanish in general.
}

An estimate of the coupling coefficient $\varpi_{S}$ is provided in the next paragraph. Regarding the term proportional to $\varpi_{\rho}$, it is expected to alter the amplitude variations of $\rho_{\theta}^{\prime}$. Such a term can be eliminated by rewriting (10) in terms of the new variable $\rho_{\theta}^{\prime} / \rho_{\eta}$ as follows:

$$
\frac{\partial}{\partial t}\left(\frac{\rho_{\theta}^{\prime}}{\rho_{\eta}}\right)+\overline{\mathbf{v}} \cdot \nabla\left(\frac{\rho_{\theta}^{\prime}}{\rho_{\eta}}\right)+\rho_{\eta}^{-1} \mathbf{v}^{\prime} \cdot \nabla \bar{\rho}_{\theta}=\varpi_{S} \frac{\rho_{S} S^{\prime}}{\rho_{\eta}},
$$

the main advantage of this formulation being that its right-hand side only involves coupling with salinity anomalies. As far as we know, this equation does not seem to have been derived before. The more classical density equation

$$
\frac{\partial \rho^{\prime}}{\partial t}+\overline{\mathbf{v}} \cdot \nabla \rho^{\prime}+\mathbf{u}^{\prime} \cdot \nabla_{h} \bar{\rho}-w^{\prime} \frac{\bar{\rho} N^{2}}{g}=0
$$

employed in most theoretical studies about Rossby waves is recovered under the following approximations: 1) neglect of the coupling term with salinity, 2) $\left|p^{\prime} / \rho^{\prime}\right| \ll$ $c_{s}^{2}$, 3) $\left\|\nabla_{h} \bar{p}\right\| /\left\|\nabla_{h} \bar{\rho}\right\| \ll c_{s}^{2}$, and 4) neglect of the spatial variations of $\rho_{\eta}$, where $N^{2}=-g / \bar{\rho}\left(\bar{\rho}_{z}-\bar{p}_{z} / c_{s}^{2}\right)$ is the classical squared Brunt-Väisälä frequency. Regarding the ratio $\rho_{\eta} / \rho_{S}$, the derivations presented in the appendix show that

$$
\frac{\rho_{s}}{\rho_{\eta}}=-\frac{(1-\kappa) C_{p}}{T} \frac{\beta_{S}}{\alpha},
$$

where $\beta_{S}$ and $\alpha$ are the haline contraction and thermal expansion coefficients, while $C_{p}$ is the isobaric heat capacity, and $\kappa=\rho \Gamma \mu_{T} / \beta_{S}$, where $\Gamma$ is the adiabatic lapse rate, and $\mu$ is the relative chemical potential (Feistel and Hagen 1995$)$. In general, $\rho_{S} / \rho_{\eta}$ will be strictly negative in most of the oceans since $\alpha$ is negative only in very confined areas of the world.

Note here that the density equation [(15)] is naturally formulated, not in terms of $\rho^{\prime}$, but rather in terms of the variable $\rho_{\theta}^{\prime}=\rho^{\prime}-p^{\prime} / c_{s}^{2}$. Of course, (15) could be made into an equation for $\rho^{\prime}$, but at the cost of additional unwanted source terms in the right-hand side involving pressure and salinity. Although it is $\rho^{\prime}$ and not $\rho_{\theta}^{\prime}$ that enters the dynamical equations [(6)], no complication is introduced, however, because the density perturbation enters (6) essentially through the hydrostatic approximation, which can be rewritten as follows:

$$
\frac{\partial p^{\prime}}{\partial z}+g \rho^{\prime}=\frac{\partial p^{\prime}}{\partial z}+\frac{1}{H_{s}} p^{\prime}+g \rho_{\theta}^{\prime}=0
$$

where $H_{s}=c_{s}^{2} / g=O(225 \mathrm{~km})$ is a scale height that is usually much larger than typical ocean depths, where we have used the values: $c_{s} \approx 1500 \mathrm{~m} \mathrm{~s}^{-1}$ and $g \approx 10$ $\mathrm{m} \mathrm{s}^{-2}$. Accordingly, (17) makes it possible to formally rewrite (6) as $\mathcal{L}\left(\mathbf{v}^{\prime}, p, \rho_{\theta}^{\prime}\right)=0$, showing that $\rho_{\theta}^{\prime}$ can be used interchangeably with $\rho^{\prime}$ in the momentum equations. 

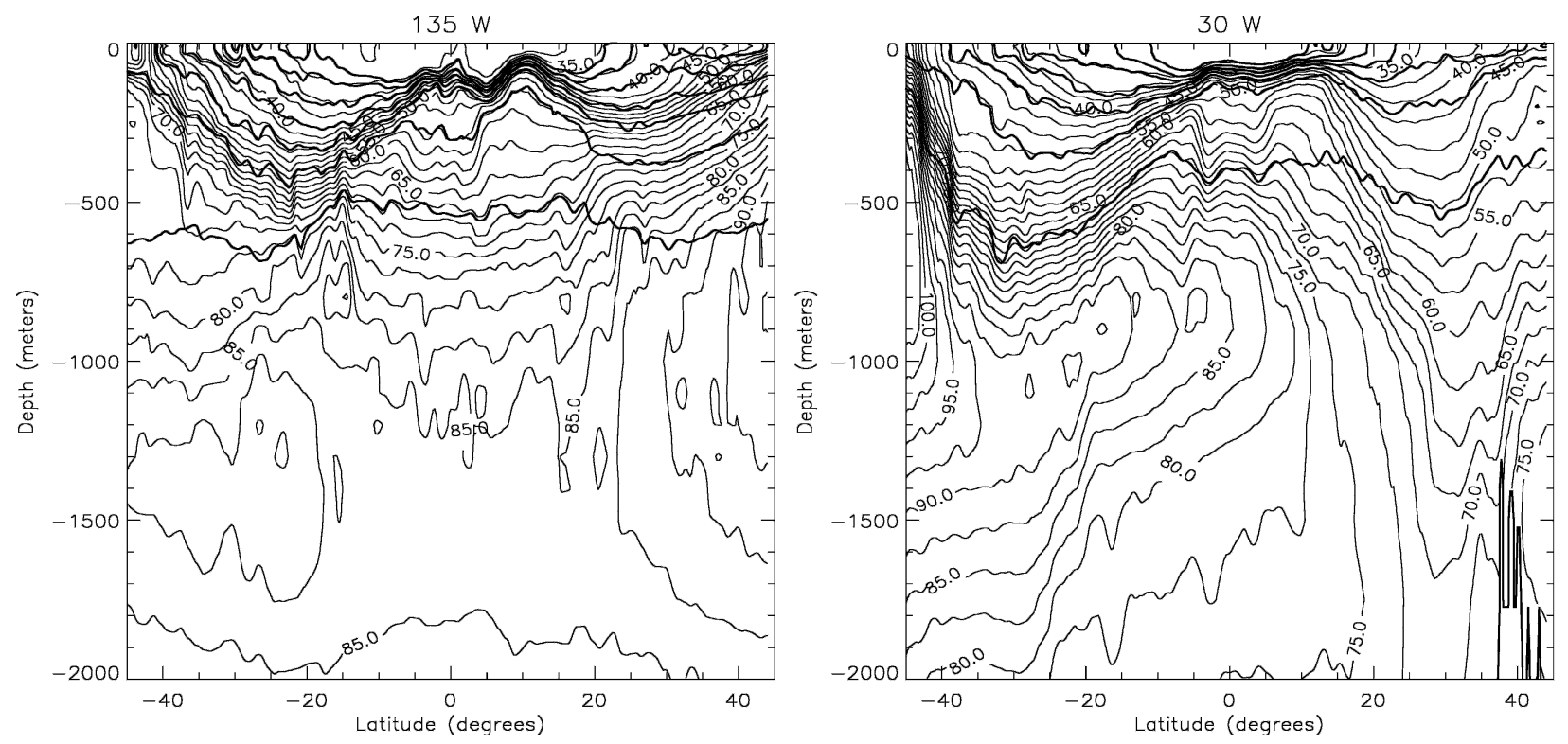

FIG. 1. Latitude-depth sections of the ratio $\left|\rho_{\eta} / \rho_{S}\right|$ for the (left) Pacific and (right) Atlantic Oceans. Superimposed as thick contours are isocontours (every $0.5 \mathrm{~kg} \mathrm{~m}^{-3}$ ) of constant $\sigma$ surfaces starting from $\sigma=27$ for the deepest one.

\section{c. Estimation of the coupling coefficient}

The unfamiliar coupling term with salinity in (15) is usually neglected in the literature. As we show in this paper, however, this coupling term, which fundamentally arises from the spatial variations of the thermodynamic coefficients of seawater, may be responsible for significant amplitude variations of $T / S$ anomalies over distances large enough for the above coefficients to undergo $O(1)$ relative variations. The order of magnitude of this coefficient can be estimated by computing the ratio $\rho_{S} / \rho_{\eta}$ from Levitus data (Boyer and Levitus 1997), the result being depicted in Fig. 1, which displays the ratio $\left|\rho_{\eta} / \rho_{S}\right|$ as a function of latitude and depth for two particular longitudes located in the Pacific and Atlantic Oceans. Overall, this ratio ranges from $O(30 \mathrm{psu} \mathrm{kg} \mathrm{K}$ $\left.\mathrm{J}^{-1}\right)$ in the surface equatorial regions to $O(100 \mathrm{psu} \mathrm{kg} \mathrm{K}$ $\mathrm{J}^{-1}$ ) at mid- and high latitudes within the thermocline. This ratio may closely follow potential density surface, while at other times it crosses them almost at right angles. This is better illustrated in Fig. 2, where the ratio is projected on various potential density surfaces for several longitudes as a function of latitude. There is little longitudinal variability in the Atlantic, but more in the Northern Pacific. Based on these figures, a tentative scaling for $\varpi_{S}$ is

$$
\varpi_{S} \approx \frac{V}{L} \frac{\Delta\left(\rho_{\eta} / \rho_{S}\right)}{\rho_{\eta} / \rho_{S}} .
$$

Using $V=10^{-2} \mathrm{~m} \mathrm{~s}^{-1}, L=10^{6} \mathrm{~m}, \Delta\left(\rho_{\eta} / \rho_{S}\right)=4$, and $\rho_{\eta} / \rho_{S}=40$ yields $\varpi_{S}=O\left(10^{-9} s^{-1}\right) \approx 1 /(30 \mathrm{yr})$. This scaling therefore suggests that the coupling with salinity anomalies becomes potentially important for propagating motions with very long decadal time scales. As a result, this effect is potentially important for the propagation of long Rossby waves at high latitudes, as well as for the density-compensated anomalies, which are the focus of the present study. Coupling effects are further discussed later in the text.

\section{Temperature and salinity signatures of the spiciness mode}

\section{a. Mathematical existence of density-compensated anomalies}

To understand the basic properties of densitycompensated anomalies, an essential first step is to understand how the existence of such anomalies can be inferred from the mathematical properties of the general equations of motion and to appreciate how these differ from classical wave solutions. In this paper, we find that the simplest linear theory for such waves requires neglecting the coupling term with salinity in (15), in which case the linearized equations of motion reduce to

$$
\begin{gathered}
\left\{\begin{array}{c}
\mathcal{L}\left(\mathbf{v}^{\prime}, p^{\prime}, \rho_{\theta}^{\prime} \rho_{\eta}\right) \\
\frac{\partial}{\partial t}\left(\frac{\rho_{\theta}^{\prime}}{\rho_{\eta}}\right)+\overline{\mathbf{v}} \cdot \nabla\left(\frac{\rho_{\theta}^{\prime}}{\rho_{\eta}}\right)+\rho_{\eta}^{-1} \mathbf{v}^{\prime} \cdot \nabla \bar{\rho}_{\theta}=0
\end{array}\right. \\
S_{t}^{\prime}+\overline{\mathbf{v}} \cdot \nabla S^{\prime}=-\mathbf{v}^{\prime} \cdot \nabla \bar{S}, \quad \text { and } \\
T^{\prime}=\frac{\beta_{S}}{\alpha} S^{\prime}+\frac{\gamma}{\alpha} p^{\prime}-\frac{\rho^{\prime}}{\rho \alpha},
\end{gathered}
$$

where we have formally expressed the momentum equations in terms of $\rho_{\theta}^{\prime} / \rho_{\eta}$ (as justified in section 2b) and added a diagnostic equation for temperature from 

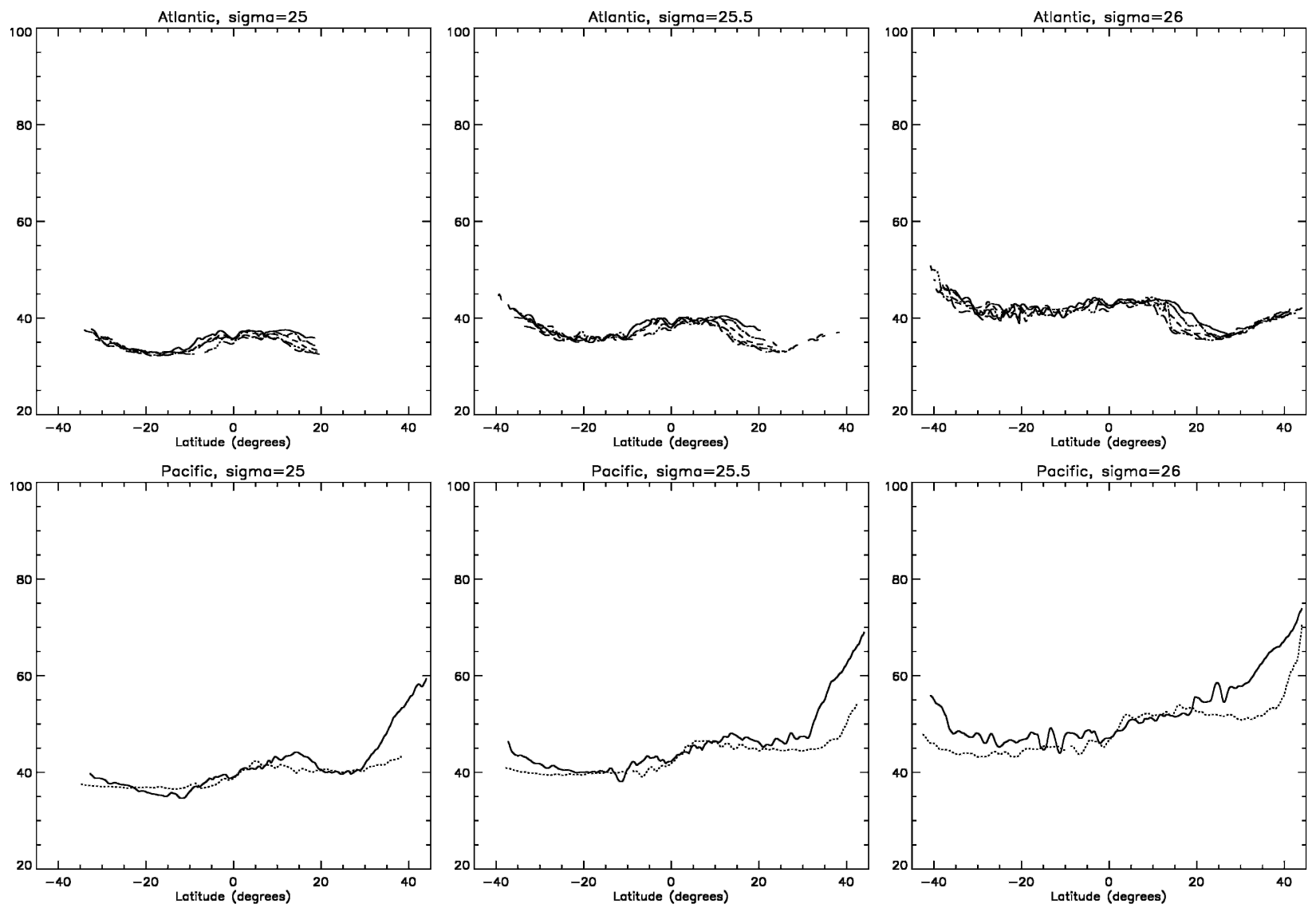

FIG. 2. Illustration of the latitudinal variations of the ratio $\left|\rho_{\eta} / \rho_{S}\right|$ projected on various $\sigma$ surfaces (from left to right) for the (top) Atlantic and the (bottom) Pacific Oceans. In the Atlantic, the four curves are associated with the longitudes $40^{\circ}, 35^{\circ}, 20^{\circ}$, and $10^{\circ} \mathrm{W}$, while in the Pacific, the two curves correspond to the longitudes $160^{\circ} \mathrm{E}$ (dotted line) and $135^{\circ} \mathrm{W}$ (solid line). Separate curves for the Atlantic are not specifically identified owing to the little longitudinal variations exhibited.

the equation of state linearized around the mean temperature, salinity, and pressure; $\rho^{\prime}=-\rho \alpha T^{\prime}+\rho \beta_{S} S^{\prime}+$ $\rho \gamma p^{\prime}$, where $\alpha, \beta_{S}$, and $\gamma$ are the classical thermal expansion, haline contraction, and isothermal compressibility coefficients.

For the present purposes, the crucial property of (18) is that it is decoupled from the salinity equation (19), which allows one to solve for $\mathbf{v}^{\prime}, p^{\prime}$, and $\rho_{\theta}^{\prime}$ independently of the equation for salinity and, hence, to assume $\mathbf{v}^{\prime}$ to be independent of $S^{\prime}$. Equation (19) is therefore a simple forced linear equation, where the forcing term is being played by the term involving $\mathbf{v}^{\prime}$, that is, the rhs of (19). Its general solution is the sum of a particular solution of the forced system plus the general solution of the homogeneous part; that is,

$$
S^{\prime}=S_{\text {wave }}+S_{\text {adv }}
$$

where $S_{\text {wave }}$ and $S_{\text {adv }}$ are respectively solutions of

$$
\frac{\partial S_{\text {wave }}}{\partial t}+\overline{\mathbf{v}} \cdot \nabla S_{\text {wave }}=-\mathbf{v}^{\prime} \cdot \nabla \bar{S} \text { and }
$$

$$
\frac{\partial S_{\text {adv }}}{\partial t}+\overline{\mathbf{v}} \cdot \nabla S_{\text {adv }}=0
$$

From (20), the corresponding general solution for temperature anomalies is as follows:

$$
T^{\prime}=T_{\mathrm{wave}}+T_{\mathrm{adv}},
$$

where

$$
\begin{aligned}
T_{\mathrm{adv}} & =\frac{\beta_{s}}{\alpha} S_{\mathrm{adv}} \text { and } \\
T_{\text {wave }} & =\frac{\beta s}{\alpha} S_{\text {wave }}+\frac{\gamma}{\alpha} p^{\prime}-\frac{\rho^{\prime}}{\rho \alpha} .
\end{aligned}
$$

These results make it clear that the system possesses two distinct types of motion: (i) wave solutions that possess a signature in the temperature, salinity, pressure, and density fields; and (ii) density-compensated solution $\left(T_{\text {adv }}, S_{\text {adv }}\right)$, which is such that $-\alpha T_{\text {adv }}+\beta_{S} S_{\text {adv }}$ $=0$, with no density and pressure signature. We thus identify $\left(T_{\text {adv }}, S_{\text {adv }}\right)$ with the temperature and salinity signatures of the so-called spiciness mode invoked by 
several authors in the recent literature (e.g., Schneider 2000).

To the extent that linear theory is able to account for the observed features, it is important to realize that both wave and density-compensated solutions must exist simultaneously in nature. The question is therefore not whether the observed seemingly advected temperature and salinity anomalies should be interpreted in terms of either active or passive dynamics, as one sometimes reads, but what is the relative importance of each kind of motions in the observed $T / S$ anomalies. Physically, the respective projection of $T / S$ anomalies into a wave part and density-compensated part is decided at the time the temperature and salinity anomalies are created, after which the active and passive components of the temperature/salinity anomalies should split and subsequently evolve along separate pathways. To decompose an arbitrary initial $\left(T^{\prime}, S^{\prime}\right)$ anomaly into its active and passive components, a natural basis is made up of the vectors $(\beta, \alpha)$ and $(\beta,-\alpha)$, yielding

$$
T_{\mathrm{adv}}=\frac{1}{2}\left(T^{\prime}+\frac{\beta_{S}}{\alpha} S^{\prime}\right) \quad \text { and } \quad S_{\mathrm{adv}}=\frac{1}{2}\left(S^{\prime}+\frac{\alpha}{\beta_{S}} T^{\prime}\right)
$$

and

$$
T_{\text {wave }}=\frac{1}{2}\left(T^{\prime}-\frac{\beta_{S}}{\alpha} S^{\prime}\right) \quad \text { and } \quad S_{\mathrm{wave}}=\frac{1}{2}\left(S^{\prime}-\frac{\alpha}{\beta_{S}} T^{\prime}\right) .
$$

One may check that (27) and (28) satisfy (24), (25), (26), and (21).

\section{SUMMARIZING REMARKS}

The mathematical demonstration for the existence of density-compensated anomalies relies on the following assumptions.

1) Nonlinearity and coupling between salinity and density anomalies is small. Indeed, both the nonlinearity and the coupling are by nature effects that would introduce a small density signature of the densitycompensated anomalies.

2) Note that, strictly speaking, density compensation as defined here only implies $\rho^{\prime}-\rho \gamma p^{\prime}=0$, which by itself does not imply that both $\rho^{\prime}$ and $p^{\prime}$ should also vanish. Thus, if we combine this result with the hydrostatic approximation, we obtain $p^{\prime}=p_{s} e^{-z / H_{e}}$ and $\rho^{\prime}=-\left[p_{s} /\left(g H_{e}\right)\right] e^{-\mathrm{z} / H_{e}}$, where $H_{e}=1 /(g \rho \gamma)$. However, if one inserts these solutions into the equations of motion, it can be established that the only consistent solution is $\rho^{\prime}=p^{\prime}=0$, which establishes, therefore, that density-compensated solutions have no pressure and density signatures at leading order.

\section{b. Characteristics of the linear spiciness mode}

Because they lack a density signature, spiciness anomalies are often assumed to behave more or less like a passive tracer. What this means physically is ambiguous, however, because the present results show that one needs to make separate statements for the temperature and salinity signatures of the spiciness mode. Indeed, (21) and (25) show that only salinity behaves like a passive tracer, but temperature does not. Specifically, if $\mathbf{x}_{2}$ and $\mathbf{x}_{1}$ are two particular points along a mean trajectory, then the consequences of (21) and (25) are that

$$
\begin{aligned}
& S_{\text {adv }}\left(\mathbf{x}_{2}\right)=S_{\text {adv }}\left(\mathbf{x}_{1}\right) \quad \text { and } \\
& T_{\text {adv }}\left(\mathbf{x}_{2}\right)=\frac{\left(\beta_{S} / \alpha\right)\left(\mathbf{x}_{2}\right)}{\left(\beta_{S} / \alpha\right)\left(\mathbf{x}_{1}\right)} T_{\text {adv }}\left(\mathbf{x}_{1}\right) .
\end{aligned}
$$

Equation (30) is interesting because it predicts that the temperature signature of the spiciness mode can be amplified or attenuated along mean trajectories as the result of the spatial variations of $\beta_{S} / \alpha$. Figure 3 shows that the surface values of the latter ratio, illustrated here for the Atlantic and Pacific Oceans, can vary significantly between the mid and high latitudes, where it reaches values of up to 3.5 and higher, and the equatorial regions where its minimum values $O(2.3-2.6)$ are reached. As a result, temperature anomalies originating from the surface mid and high latitudes and aiming at the surface equatorial regions can only decrease over time on average, in the absence of other effects (i.e., diabatism, nonlinear effects, coupling with wave modes). For instance, taking an anomaly created around $40^{\circ}(\mathrm{N}$ or $\mathrm{S})$, where $\beta_{S} / \alpha \approx 3$, yields an attenuation factor of about $2.3 / 3 \approx 0.77$ upon reaching the equator. This is a significant factor, considering that other effects such as diffusion are not considered here.

It is important to note here that a mean trajectory cannot link the surface midlatitudes to the surface equatorial regions without crossing various isopycnal surfaces, or equivalently without possessing a significant diapycnal component, most likely in the surface mixed layer. This implicitly supposes nonconservative effects not accounted for by the present theory. For this reason, it is important to distinguish between changes of $\beta_{S} / \alpha$ taking place across isopycnals, for which the present theory is incomplete, from changes occurring purely along isopycnals. To that end, Fig. 4 shows the function $\beta_{S} / \alpha$ as a function of latitude and depth for two particular longitudes, along with a number of isopycnal surfaces. It shows that $\beta_{S} / \alpha$ usually increases with depth to reach a maximum of $O(6-7)$ near $1000-1500 \mathrm{~m}$, that is, within the thermocline, to then decrease. On the other hand, $\beta_{S} / \alpha$ is often found to vary little over significant portions of a given isopycnal surface with more significant variations occurring over localized regions. In general, however, variations of $\beta_{S} / \alpha$ along isopycnal surfaces are often much less important than those taking place across isopycnals. To make this more accurate, we depicted in Fig. 5 the value of $\beta_{S} / \alpha$ projected on various potential density surfaces of interest as a function of latitude for several longitudes. As an ex- 

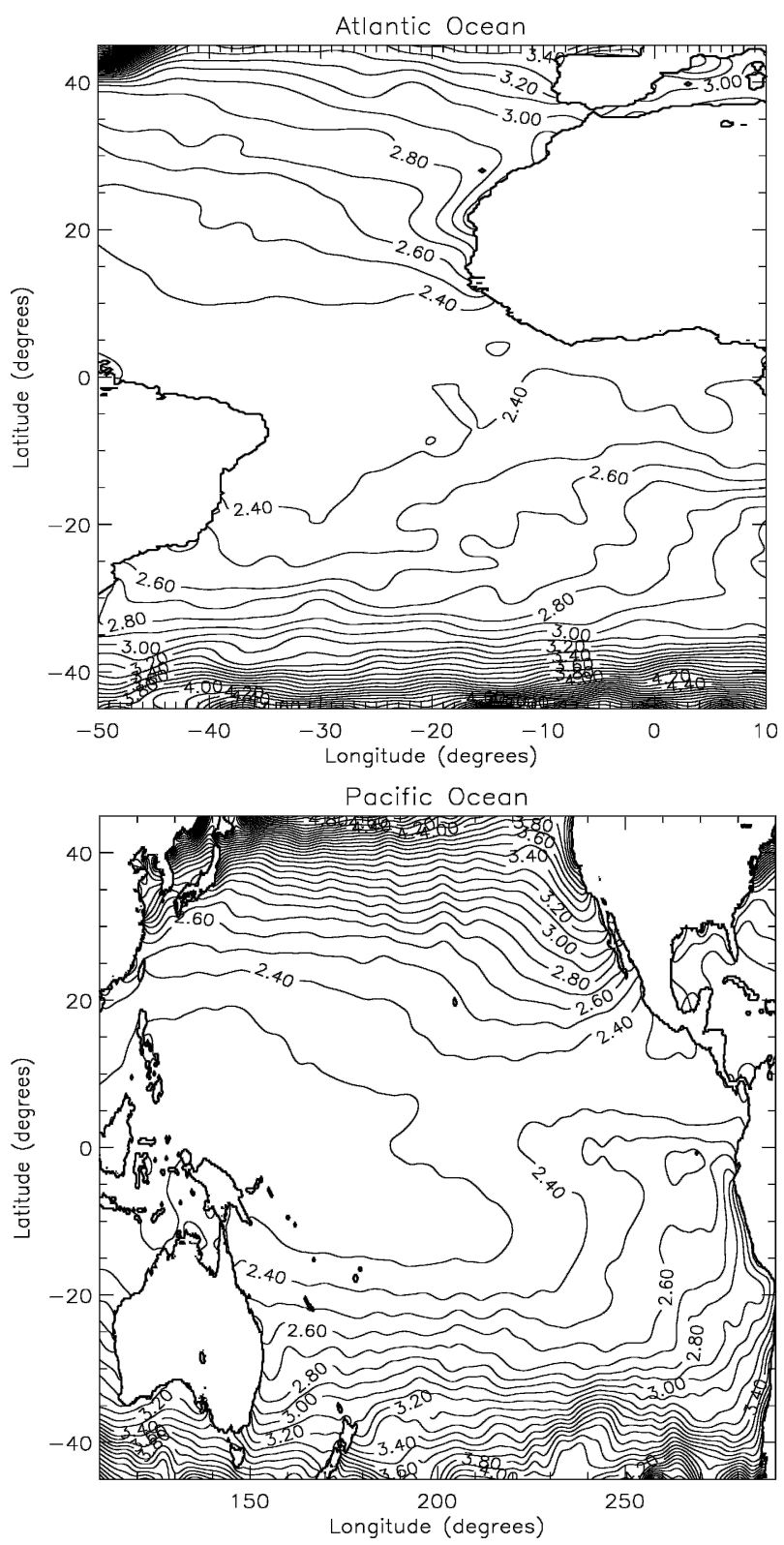

FIG. 3. Surface values of the ratio $\beta_{S} / \alpha$ in the (top) Atlantic and (bottom) Pacific Oceans.

ample of possible behavior along a given potential density surface, consider an anomaly at $40^{\circ} \mathrm{S}$ on the $\sigma=26$ density surface where $\beta_{S} / \alpha \approx 3$ and bring it to the equator where $\beta_{S} / \alpha \approx 3.2$. This time, the "attenuation" factor is only $3.2 / 3 \approx 1.067$, which is, in fact, a slight amplification. If we start instead from $40^{\circ} \mathrm{N}$ where $\beta_{S} / \alpha \approx$ 3.4 , then the attenuation factor is this time $3.2 / 3.4 \approx$ 0.94 , which is small. The examination of the other panels show that, in general, amplification or attenuation factors along isopycnals can only be small in the Atlantic. Stronger attenuation factors appear to be possible in the Pacific, however, for anomalies created in the
North Pacific. For instance, starting from $40^{\circ} \mathrm{N}$ where $\beta_{S} / \alpha \approx 4$ on the $\sigma=25$ surface up to the equator where $\beta_{S} / \alpha \approx 2.9$ yields an attenuation factor of $2.9 / 4=0.72$, which is this time quite significant.

In view of the above, it appears clearly that most of what makes up the above-computed attenuation factor of the ratio $\beta_{S} / \alpha$ from the surface midlatitudes to the equatorial surface actually comes from the diapycnal variations of $\beta_{S} / \alpha$ rather than from its isopycnal variations. For our computation to be meaningful, then, it is necessary to assume that the diabatically induced amplitude variations of the ratio $T^{\prime} / S^{\prime}$ can be somehow computed separately from the adiabatic ones that are the focus of this paper. It is too early to say whether this is justified or not, as addressing this issue requires a more sophisticated model, which is beyond the scope of this preliminary study.

\section{Robustness and accuracy issues}

The prediction achieved in the previous section relies, apart from the assumption of linearity, on the choice of density and salinity as the prognostic variables and on the neglect of the coupling between density and salinity. As seen previously, the time scale of the coupling was estimated to be $O(10 \mathrm{yr})$, which scales as the time scale required for the water masses considered to travel over the large distances of interest here [e.g., see Lazar et al. (2002) and Rothstein et al. (1998) for estimates in the Atlantic and Pacific Oceans, respectively]. Therefore, such a coupling potentially introduces an uncertainty about the previous prediction, which the following paragraphs seek to clarify and quantify.

\section{a. Comparison with density/spiciness formulation}

Since the choice of the main prognostic variables is not unique, it is of interest to test the robustness of the previous prediction to a different choice than density and salinity as prognostic variables. To that end, we shall seek to replace the coupled density/salinity equations (15)-(8) by equivalent coupled equations for density $\rho=\rho(S, \eta, p)$ and an arbitrary function of state $\xi(S$, $\eta, p)$, which encompasses the previous case for $\xi=S$. To obtain the sought-for coupled equations for $\rho^{\prime}$ and $\xi^{\prime}$ first requires linearizing $\rho$ and $\xi$ around their mean values; namely,

$$
\begin{gathered}
\rho^{\prime}-\frac{1}{c_{s}^{2}} p^{\prime}=\rho_{\theta}^{\prime}=\rho_{\eta} \eta^{\prime}+\rho_{S} S^{\prime} \quad \text { and } \\
\xi^{\prime}-\xi_{p} p^{\prime}=\xi_{\theta}^{\prime}=\xi_{\eta} \eta^{\prime}+\xi_{S} S^{\prime} .
\end{gathered}
$$

By combining (7) and (8) with (31) and (32), the following evolution equations for $\rho^{\prime}$ and $\xi^{\prime}$ are eventually obtained:

$$
\frac{\partial \rho_{\theta}^{\prime}}{\partial t}+\overline{\mathbf{v}} \cdot \nabla \rho_{\theta}^{\prime}+\mathbf{v}^{\prime} \cdot\left(\nabla \bar{\rho}-\frac{1}{c_{s}^{2}} \nabla \bar{p}\right)=\varpi_{1} \rho_{\theta}^{\prime}+\varpi_{2} \xi_{\theta}^{\prime}
$$



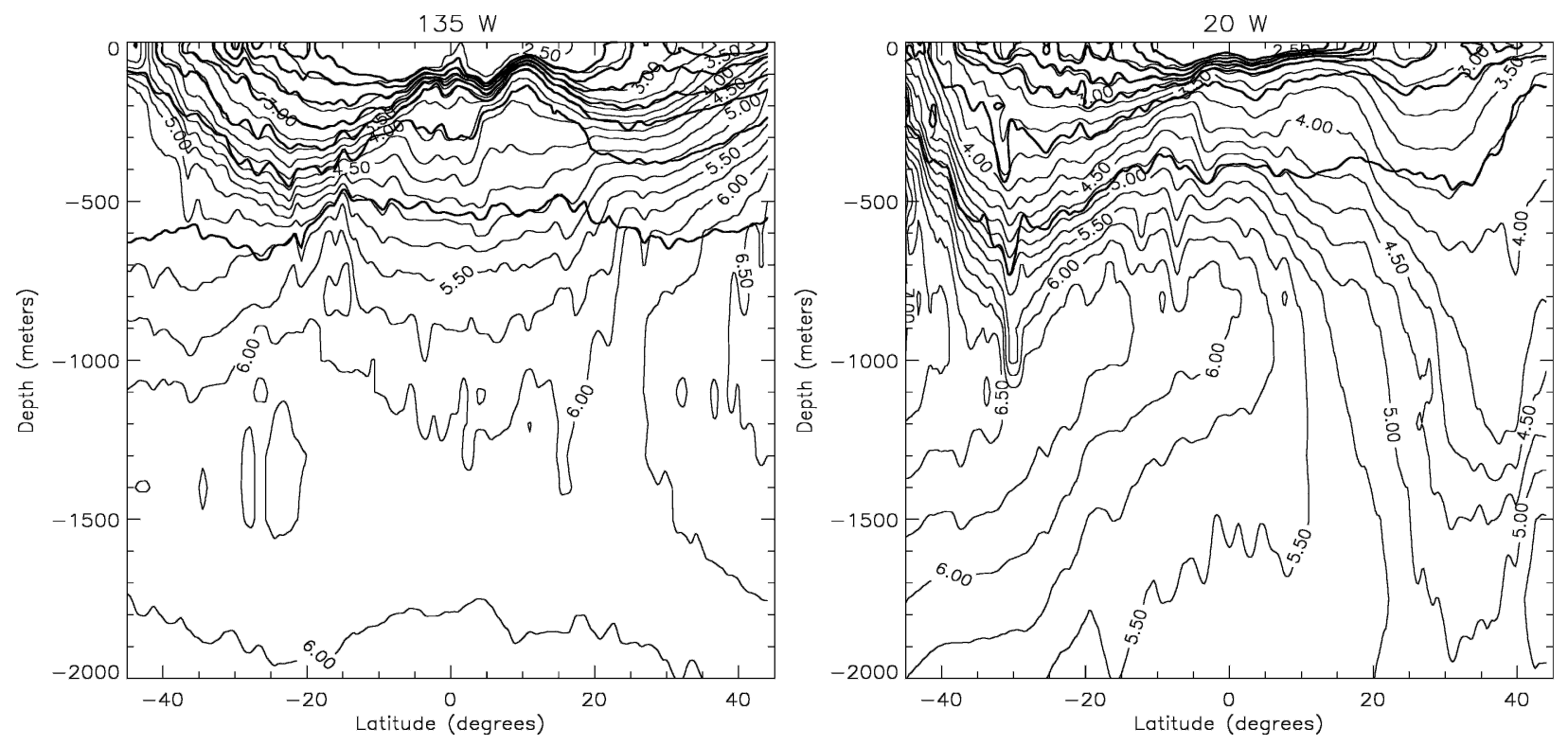

FIG. 4. As in Fig. 1 but for the ratio $\beta_{S} / \alpha$.

$\frac{\partial \xi_{\theta}^{\prime}}{\partial t}+\overline{\mathbf{v}} \cdot \nabla \xi_{\theta}^{\prime}+\mathbf{v}^{\prime} \cdot\left(\nabla \bar{\xi}-\xi_{p} \nabla \bar{p}\right)=\varpi_{3} \rho_{\theta}^{\prime}+\varpi_{4} \xi_{\theta}^{\prime}$,

where the coefficients $\varpi_{i}, i=1, \ldots, 4$ are given by the expressions

$$
\begin{aligned}
\varpi_{1} & =\left(\xi_{S} \overline{\mathbf{v}} \cdot \nabla \rho_{\eta}-\xi_{\eta} \overline{\mathbf{v}} \cdot \nabla \rho_{S}\right) / J, \\
\varpi_{2} & =\left(\rho_{\eta} \overline{\mathbf{v}} \cdot \nabla \rho_{S}-\rho_{S} \overline{\mathbf{v}} \cdot \nabla \rho_{\eta}\right) / J, \\
\varpi_{3} & =\left(\xi_{S} \overline{\mathbf{v}} \cdot \nabla \xi_{\eta}-\xi_{\eta} \overline{\mathbf{v}} \cdot \nabla \xi_{S}\right) / J, \\
\varpi_{4} & =\left(\rho_{\eta} \overline{\mathbf{v}} \cdot \nabla \xi_{S}-\rho_{S} \overline{\mathbf{v}} \cdot \nabla \xi_{\eta}\right) / J, \quad \text { and } \\
J & =\rho_{\eta} \xi_{S}-\rho_{S} \xi_{\eta} .
\end{aligned}
$$

Equations (33) and (34) and the expressions (35)-(38) make it clear that a wide variety of coupled prognostic equations is possible. Now, the magnitude of the coupling terms, namely, the terms proportional to $\varpi_{2}$ and $\varpi_{3}$ in (33) and (34), depends in general upon the particular choice of $\xi(S, \eta, p)$. From a theoretical viewpoint, the question of interest is whether there exists an "optimal" way to define the function of state $\xi$, which minimizes the coupling effects. If so, such a choice would provide the greatest accuracy for the leadingorder approximations for $S_{\text {adv }}$ and $T_{\text {adv }}$. Mathematically, however, we lack a clear way to define a suitable optimization problem for $\xi$ that would guarantee that the desired result has been achieved. For this reason, we resort to physical intuition to postulate that the optimal choice for $\xi$ is probably a form of spiciness [e.g., see Flament (2002) for a recent review of the concept]. Indeed, spiciness is widely regarded as being more "orthogonal" to density than salinity, and hence likely to be less coupled to density than salinity. In any case, the choice of spiciness is justified if only by the present context, which is to understand the dynamics of what is referred to in the literature as the "spiciness mode." To proceed, we define $\xi$ by

$$
\xi_{S}=\lambda \rho_{S} \quad \text { and } \quad \xi_{\eta}=-\lambda \rho_{\eta},
$$

where $\lambda$ is an integrating factor required for the following compatibility condition to be satisfied:

$$
\frac{\partial}{\partial S}\left(\lambda \frac{\partial \rho}{\partial \eta}\right)+\frac{\partial}{\partial \eta}\left(\lambda \frac{\partial \rho}{\partial S}\right)=0
$$

The knowledge of $\lambda$ is unimportant for the present study, and therefore the details of how to obtain the latter are not given here. The study of Flament (2002) entirely neglects it. By using (40), the expressions for (35)-(38) become

$$
\begin{aligned}
& \varpi_{1}=\overline{\mathbf{v}} \cdot \nabla \ln \sqrt{\left|\rho_{\eta} \rho_{S}\right|}, \\
& \varpi_{2}=\frac{1}{\lambda} \overline{\mathbf{v}} \cdot \nabla \ln \sqrt{\mid \frac{\rho_{S} \mid}{\rho_{\eta} \mid}}=\frac{\varpi_{S}}{2 \lambda}, \\
& \varpi_{3}=\lambda \overline{\mathbf{v}} \cdot \nabla \ln \sqrt{\mid \frac{\rho_{S}}{\rho_{\eta} \mid}}=\frac{\lambda \varpi_{S}}{2}, \text { and } \\
& \varpi_{4}=\overline{\mathbf{v}} \cdot \nabla \ln \sqrt{\lambda^{2}\left|\rho_{\eta} \rho_{S}\right|} .
\end{aligned}
$$

To go further, we chose to remove the pseudodamping terms (those proportional to $\varpi_{1}$ and $\varpi_{4}$ ) in (33) and (34) by introducing the two following variables:

$$
\tilde{\rho}_{\theta}^{\prime}=\frac{\rho_{\theta}^{\prime}}{\sqrt{\left|\rho_{S} \rho_{\eta}\right|}} \text { and } \tilde{\xi}_{\theta}^{\prime}=\frac{\xi_{\theta}^{\prime}}{\lambda \sqrt{\left|\rho_{S} \rho_{\eta}\right|}}
$$



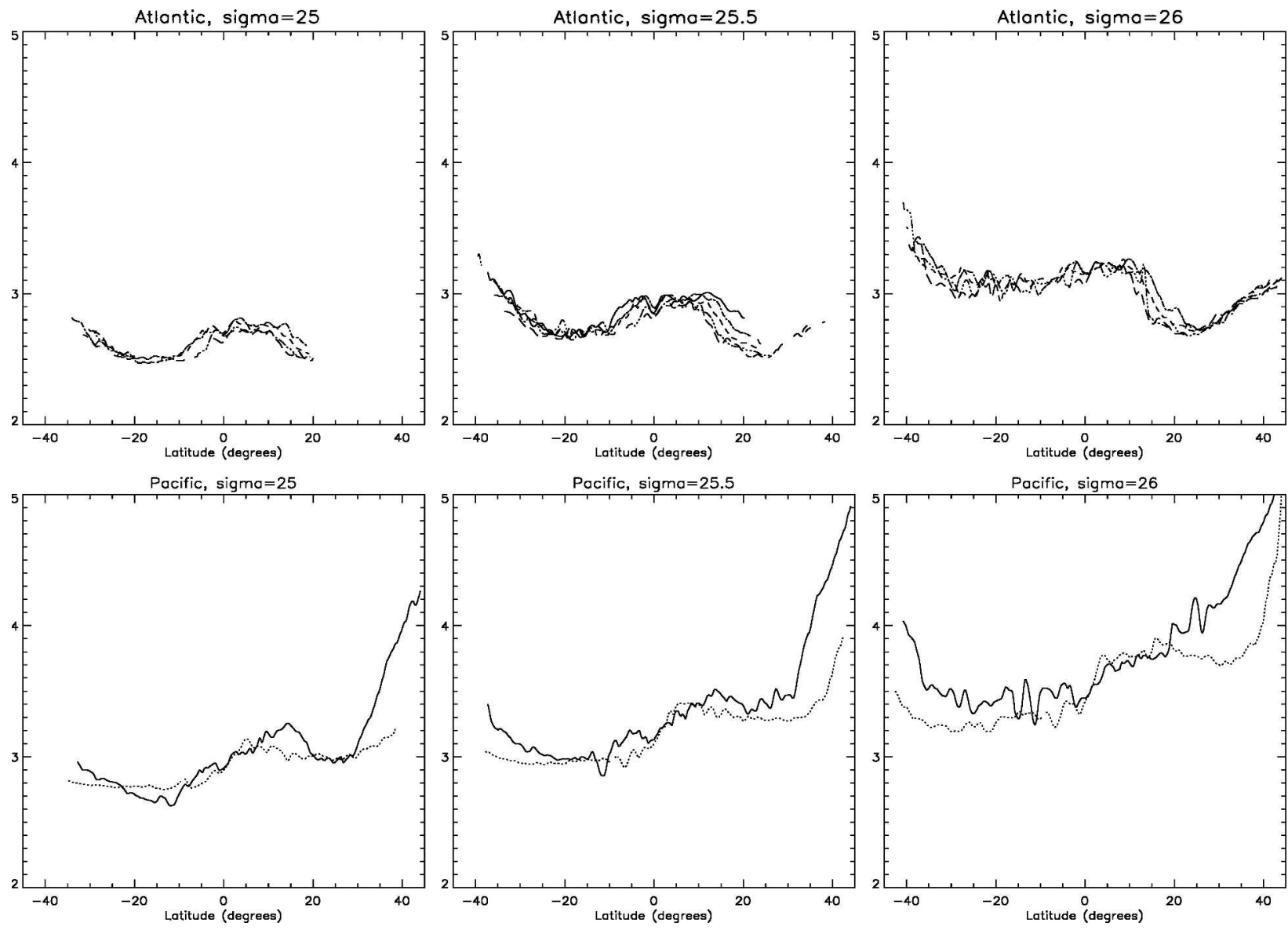

FIG. 5. As in Fig. 2 but for the ratio $\beta_{S} / \alpha$.

so that, after some manipulation, (33)-(34) become

$\frac{\partial \tilde{\rho}_{\theta}^{\prime}}{\partial t}+\overline{\mathbf{v}} \cdot \nabla \tilde{\rho}_{\theta}^{\prime}+\left(\left|\rho_{S} \rho_{\eta}\right|\right)^{-1 / 2} \mathbf{v}^{\prime} \cdot\left(\nabla \bar{\rho}-\frac{1}{c_{s}^{2}} \nabla \bar{p}\right)=\frac{\varpi_{S}}{2} \tilde{\xi}_{\theta}^{\prime}$

and

$\frac{\partial \tilde{\xi}_{\theta}^{\prime}}{\partial t}+\overline{\mathbf{v}} \cdot \nabla \tilde{\xi}_{\theta}^{\prime}+\left(\lambda^{2}\left|\rho_{S} \rho_{\eta}\right|\right)^{-1 / 2} \mathbf{v}^{\prime} \cdot\left(\nabla \bar{\xi}-\xi_{p} \nabla \bar{p}\right)=\frac{\varpi_{S}}{2} \tilde{\rho}_{\theta}^{\prime}$

The main indication that the coupling between (47) and (48) is less important than between (15) and (8) is that the coupling coefficient is reduced by a factor of 2 in the former in comparison with that in (15). Another interesting property that might be relevant, although exactly how is unclear, is the perfect symmetry of the coupling terms. In any case, constructing a leading-order theory similarly as before by neglecting the coupling terms in (47) and (48) (i.e., the rhs), and proceeding similarly as in section 3 yields the following general solution for $\xi_{\theta}^{\prime}$ and $\tilde{\xi}_{\theta}^{\prime}$ :

$$
\tilde{\xi}_{\theta}^{\prime}=\xi_{\mathrm{adv}}+\xi_{\mathrm{wave}} \quad \text { and } \quad \xi_{\theta}^{\prime}=\lambda \sqrt{\left|\rho_{\eta} \rho_{S}\right|}\left(\xi_{\mathrm{adv}}+\xi_{\mathrm{wave}}\right)
$$

where this time it is $\xi_{\text {adv }}$ which satisfies the "passive tracer" equation:

$$
\frac{\partial \xi_{\mathrm{adv}}}{\partial t}+\overline{\mathbf{v}} \cdot \nabla \xi_{\mathrm{adv}}=0
$$

Regarding the temperature and salinity signatures, they are easily shown to be given by

$$
S^{\prime}=\frac{\rho_{\theta}^{\prime}+\xi_{\theta}^{\prime} / \lambda}{2(1-\kappa) \rho \beta_{S}} \quad \text { and } \quad T^{\prime}=\frac{\xi_{\theta}^{\prime} / \lambda-(1-2 \kappa) \rho_{\theta}^{\prime}}{2(1-\kappa) \rho \alpha}+\Gamma p^{\prime}
$$

so that the corresponding signatures for the spiciness mode are given by

$$
S_{\mathrm{adv}}=\frac{\sqrt{\left|\rho_{\eta} \rho_{S}\right|}}{2(1-\kappa) \rho \beta_{S}} \xi_{\mathrm{adv}}=\frac{1}{2} \sqrt{\left|\frac{\rho_{\eta}}{\rho_{S}}\right|} \xi_{\mathrm{adv}}
$$

and

$$
T_{\mathrm{adv}}=\frac{1}{2} \frac{\beta_{S}}{\alpha} \sqrt{\left|\frac{\rho_{\eta}}{\rho_{S}}\right|} \xi_{\mathrm{adv}}
$$

by using the result $(1-\kappa) \rho \beta_{S}=\rho_{S}$ from (A5). 

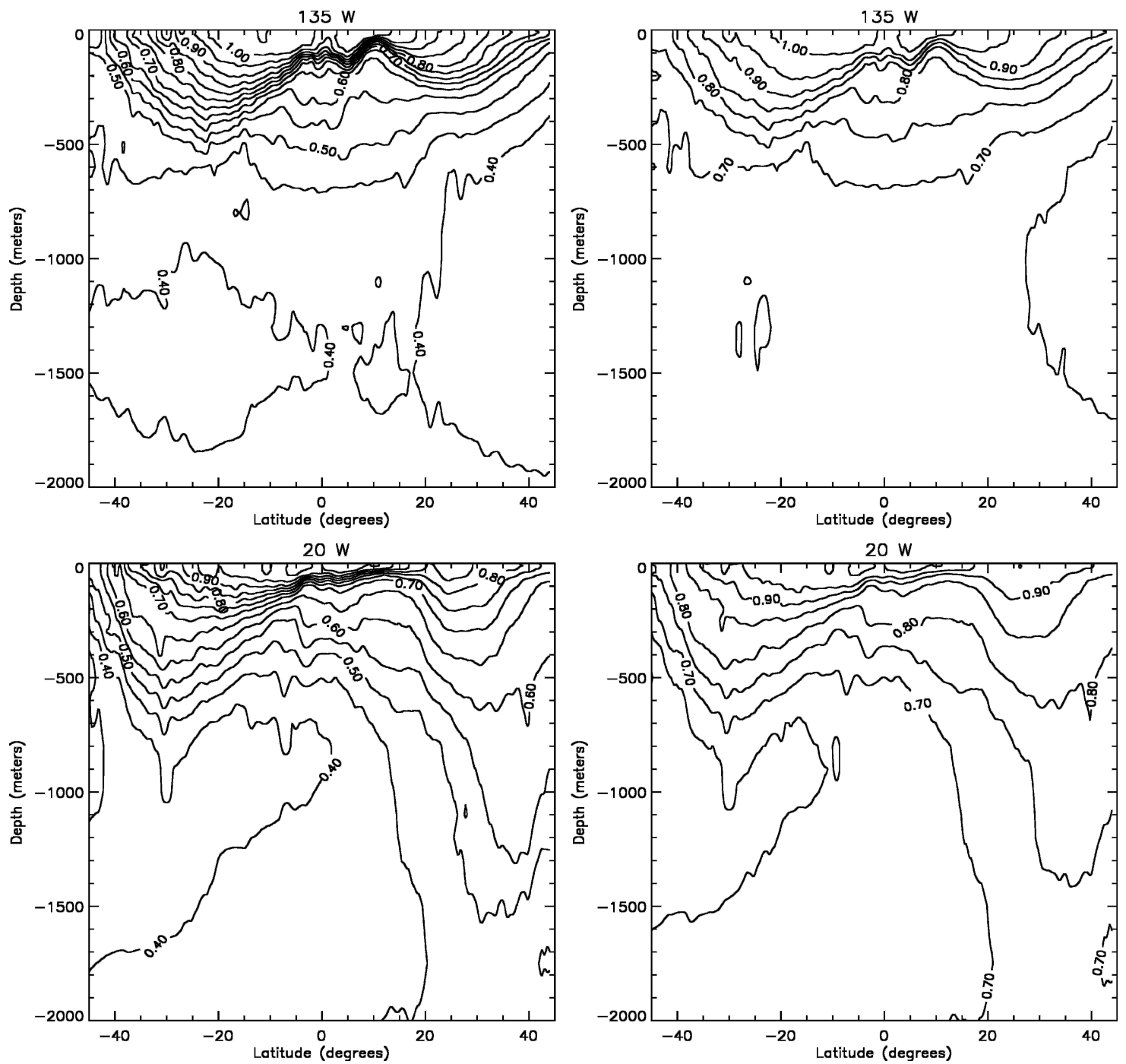

FIG. 6. Latitude-depth sections of the ratio $T_{\text {adv }}\left(\mathbf{x}_{2}\right) / T_{\text {adv }}\left(\mathbf{x}_{1}\right)$ at (top) $135^{\circ} \mathrm{W}$ computed from the (left) leading-order density/salinity theory and (right) density/spiciness theory, assuming that $\mathbf{x}_{1}$ is located at the equatorial surface. In other words, $\mathbf{x}_{2}=\left(135^{\circ} \mathrm{W}, y, z\right)$ and $\mathbf{x}_{1}=\left(135^{\circ} \mathrm{W}, 0,0\right)$. (bottom) Same as above panels but in the Atlantic Ocean along $20^{\circ} \mathrm{W}$.

Now, (52)-(53) show that $T_{\text {adv }} / S_{\text {adv }}=\beta_{S} / \alpha$ exactly as when using density and salinity as prognostic variables; however, the predictions for the respective evolution of temperature and salinity anomalies along mean streamlines are somewhat different. Specifically, if we consider two points $\mathbf{x}_{1}$ and $\mathbf{x}_{2}$ along the same mean streamline, the new prediction is that

$$
\begin{aligned}
& S_{\text {adv }}\left(\mathbf{x}_{2}\right)=\frac{\sqrt{\left|\rho_{\eta} / \rho_{S}\right|}\left(\mathbf{x}_{2}\right)}{\sqrt{\left|\rho_{\eta} / \rho_{S}\right|}\left(\mathbf{x}_{1}\right)} S_{\text {adv }}\left(\mathbf{x}_{1}\right) \text { and } \\
& T_{\text {adv }}\left(\mathbf{x}_{2}\right)=\frac{\left(\beta_{S} / \alpha\right)\left(\mathbf{x}_{2}\right)}{\left(\beta_{S} / \alpha\right)\left(\mathbf{x}_{1}\right)} \frac{\sqrt{\left|\rho_{\eta} / \rho_{S}\right|}\left(\mathbf{x}_{2}\right)}{\sqrt{\left|\rho_{\eta} / \rho_{S}\right|}\left(\mathbf{x}_{1}\right)} T_{\text {adv }}\left(\mathbf{x}_{1}\right) .
\end{aligned}
$$

Equations (54) and (55) are the counterparts of (29) and (30); they show that the previous prediction for the attenuation factors are modified by the new term $\sqrt{\left|\rho_{\eta} / \rho_{S}\right|}$. In this theory, salinity anomalies are no longer conserved along the mean streamlines. To assess the impact of this correction, we depicted the ratio $T_{\text {adv }}\left(\mathbf{x}_{2}\right) /$ $T_{\text {adv }}\left(\mathbf{x}_{1}\right)$ predicted by the two theories in Fig. 6 (left panels versus right panels) assuming that $\mathbf{x}_{1}$ is located on the equatorial surface and $\mathbf{x}_{2}$ arbitrary for a given longitude. This correction significantly reduces the attenuation factors previously found and acts in making density-compensated temperature anomalies more conserved along streamlines. For instance, the ratio in the new theory at $40^{\circ} \mathrm{N}$ is about 0.85 , instead of $0.7-0.75$ with the previous theory, at $135^{\circ} \mathrm{W}$. The impact on density-compensated salinity anomalies is illustrated in Fig. 7 , which depicts the ratio $S_{\text {adv }}\left(\mathbf{x}_{2}\right) / S_{\text {adv }}\left(\mathbf{x}_{1}\right)$ for two selected longitudes, assuming again that $\mathbf{x}_{1}$ is located on the equatorial surface. An example of average surface 

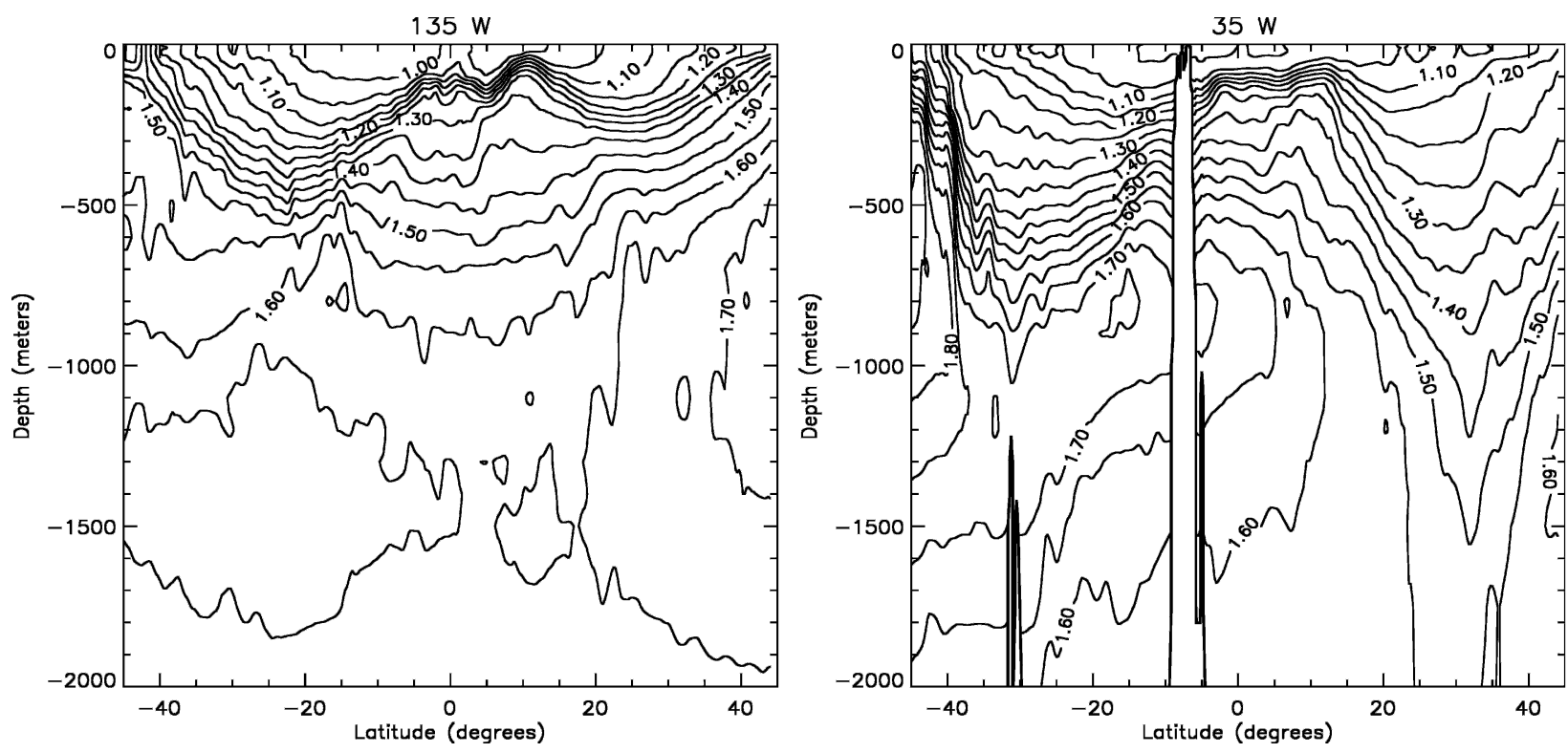

FIG. 7. Latitude-depth sections of the ratio $S_{\text {adv }}\left(\mathbf{x}_{2}\right) / S_{\text {adv }}\left(\mathbf{x}_{1}\right)$ computed as in the previous figure by assuming $\mathbf{x}_{1}$ is located at the equatorial surface, for a particular depth-latitude section in the (left) Pacific and (right) Atlantic.

amplification factor as defined previously is about 1.21.25 at $40^{\circ} \mathrm{N}, 135^{\circ} \mathrm{W}$ in the Pacific Ocean and about 1.15 at $40^{\circ} \mathrm{N}, 35^{\circ} \mathrm{W}$ in the Atlantic Ocean. Such factors are quite significant and should be measurable.

\section{b. Is the density/spiciness formulation really more accurate?}

The prediction that $T^{\prime} / S^{\prime}=\beta_{S} / \alpha$ along the mean streamlines is simply the consequence of the anomalies being density compensated and is independent of the particular choice of prognostic variables. The latter is not true, however, of the prediction for the respective evolution of the temperature and salinity anomalies. Nevertheless, regardless of the choice of prognostic variables, it is possible to state that there must exist a position-dependent function $A$ such that between two arbitrary points $\mathbf{x}_{1}$ and $\mathbf{x}_{2}$ along a given streamline one must have

$$
\begin{aligned}
T^{\prime}\left(\mathbf{x}_{2}\right) & =\frac{\left(\beta_{S} / \alpha\right)\left(\mathbf{x}_{2}\right) A\left(\mathbf{x}_{2}\right)}{\left(\beta_{S} / \alpha\right)\left(\mathbf{x}_{1}\right) A\left(\mathbf{x}_{1}\right)} T^{\prime}\left(\mathbf{x}_{1}\right) \text { and } \\
S^{\prime}\left(\mathbf{x}_{2}\right) & =\frac{A\left(\mathbf{x}_{2}\right)}{A\left(\mathbf{x}_{1}\right)} S^{\prime}\left(\mathbf{x}_{1}\right) .
\end{aligned}
$$

As seen previously, the function $A$ depends on the particular choice of prognostic variables, with $A=1$ for the density/salinity formulation, and $A=\left|\rho_{S} / \rho_{\eta}\right|^{1 / 2}$ for the density/spiciness formulation. Obviously, each different choice of prognostic variables will yield a different result for $A$. It is therefore important to determine which particular choice of prognostic variables yields the most accurate prediction for $A$ ?

To answer this nontrivial question, we formally estimate the next order correction to $A$ from the density/ salinity formulation, which is expected to improve upon the leading-order theory and establish that the form thus predicted for $A$ is consistent, if only qualitatively, with the leading-order prediction of the density/ spiciness formulation. We believe that this establishes that the leading-order density/spiciness formulation is more accurate than its density/salinity counterpart.

To proceed, we use a classical perturbation approach in terms of the (assumed) small coupling parameter $\varpi_{S}$. Specifically, we write $\varpi_{S}$ under the form

$$
\varpi_{S}=\varepsilon \varpi_{S}^{*},
$$

where $\varpi_{S}^{*}$ is of order unity and $\varepsilon$ is a dimensionless measure of the smallness of $\varpi_{S}$. Let us also write $S_{\text {adv }}=$ $\Delta S_{0} Q_{\text {adv }}$, where $\Delta S_{0}$ is a constant having the physical dimension of salinity so that $Q_{\text {adv }}$ is dimensionless and satisfies $\partial_{t} Q_{\mathrm{adv}}+\overline{\mathbf{v}} \cdot \boldsymbol{\nabla} Q_{\mathrm{adv}}=0$. The salinity, temperature, density, and pressure signatures of the spiciness mode are sought as the following series expansions:

$$
\begin{aligned}
S^{\prime} & =S_{\mathrm{adv}}=\Delta S_{0} Q_{\mathrm{adv}}(x, y, z, t)\left[1+\varepsilon s_{0}(x, y, z)+O\left(\varepsilon^{2}\right)\right], \\
T^{\prime} & =T_{\mathrm{adv}}=\frac{\beta_{S}}{\alpha} S_{\mathrm{adv}}=\frac{\beta_{S} \Delta S_{0}}{\alpha} Q_{\mathrm{adv}}(x, y, z, t)\left[1+\varepsilon S_{0}(x, y, z)+O\left(\varepsilon^{2}\right)\right], \quad \text { and } \\
\left(\mathbf{v}^{\prime}, \rho_{\theta}^{\prime} / \rho_{\eta}, p^{\prime}\right) & =\varepsilon Q_{\mathrm{adv}}(x, y, z, t)\left[\mathbf{v}_{0}(x, y, z), \rho_{0}(x, y, z), p_{0}(x, y, z)\right]+O\left(\varepsilon^{2}\right) .
\end{aligned}
$$


From (59), it is clear that the required expression for $A$ is as follows:

$$
A(x, y, z)=1+\varepsilon s_{0}(x, y, z)+\left[O\left(\varepsilon^{2}\right)\right] .
$$

Note that at leading order the spiciness mode has no density, pressure, and velocity signatures, but this is no longer true at the order $O(\varepsilon)$. Now, inserting (58) and (60) into (6), (8), and (15) leads to the following (formal) system for $\rho_{0}, p_{0}$, and $\mathbf{v}_{0}$ :

$$
\begin{gathered}
\mathcal{L}\left(\mathbf{v}_{0}, \rho_{0}, p_{0}\right)=0, \\
\overline{\mathbf{v}} \cdot \nabla \rho_{0}+\rho_{\eta}^{-1} \mathbf{v}_{0} \cdot \nabla \bar{\rho}_{\theta}=\frac{\rho_{S} \varpi_{S}^{*}}{\rho_{\eta}} \Delta S_{0}, \quad \text { and } \\
\overline{\mathbf{v}} \cdot \nabla s_{0}+\mathbf{v}_{0} \cdot \nabla \bar{S}=0 .
\end{gathered}
$$

Mathematically, the coupled system (62)-(64) is a timeindependent forced linear system, where the forcing term is played by the rhs of (63). For the present purposes, we do not need to solve for the general solution of (62)-(64), which would be difficult anyway. Let us simply point out that $\varpi_{s}$ and hence the right-hand side of (63) is a function of $\rho_{S} / \rho_{\eta}$, which implies that formally $s_{0}=s_{0}\left(\rho_{S} / \rho_{\eta} ; \ldots\right)$, and hence $A=A\left(\rho_{S} / \rho_{\eta}, \ldots\right)$ as well. This dependence upon $\rho_{S} / \rho_{\eta}$ is precisely that exhibited by the function $A$ predicted by the density/spiciness theory. This suggests, therefore, that the leading-order density/spiciness theory is able to capture some of the higher-order corrections of the density/salinity theory, which implies that it is therefore the most accurate of the two leading-order theories. This result confirms that the coupling of density and salinity anomalies is not negligible on the gyre scale, but that it can perhaps be neglected between density and spiciness anomalies. Note that the next-order correction for the density/ spiciness formulation would also depend on $\rho_{S} / \rho_{\eta}$, and would simply confirm that $A$ must depend on $\rho_{S} / \rho_{\eta}$ regardless of which order the respective theories are pushed to.

\section{Discussion and conclusions}

In this paper, we developed a theoretical framework to study the physical and thermodynamical processes controlling the adiabatic amplitude variations of largescale propagating temperature and salinity anomalies in a realistic ocean. Such anomalies are now widely assumed to fall broadly into two main categories: waves and instabilities possessing a density/pressure signature and passively advected density-compensated anomalies with no density/pressure signature (Schneider et al. 1999; Lazar et al. 2001). In this paper, we point out that the existence of strictly compensated anomalies is only possible under the approximation that the anomalies obey linear and uncoupled thermodynamics equations. In other words, actual "density compensated" anomalies must be expected to possess a signature, if only small, in the pressure and density fields. Such approxi- mations are commonly made in the context of deriving amplitude equations for waves in the context of WKB theory, for instance, which establishes that amplitudes variations of waves are primarily controlled by the divergence of the group velocity. In this paper, the focus is on understanding what controls the amplitude evolution of density-compensated anomalies, for which there is no general methodology available, by retaining the assumption of linearity but not necessarily that of uncoupled thermodynamics.

The need to study the amplitude variations of density-compensated temperature and salinity anomalies may seem to be strange at first because their passive character is often assumed to imply that such anomalies are conserved along the mean streamlines. In this respect, the "conservativeness" of passive advection is often opposed to the "decaying" character of wave dispersion. However, as shown extensively in this paper, the main issue with density-compensated $T / S$ anomalies is that they need to maintain the equality $T^{\prime} / S^{\prime}=\beta_{S} / \alpha$ along the mean streamlines to maintain density compensation. As a result, the conservation of $T^{\prime}$ and $S^{\prime}$ along mean streamlines depends largely on whether $\beta_{S} / \alpha$ is also conserved along the latter. Observational evidence from available hydrological data, however, demonstrates that $\beta_{S} / \alpha$ may undergo significant spatial variations both horizontally and vertically, but it important to note that isopycnal variations of $\beta_{S} / \alpha$ are often found to be much less important than cross-isopycnal variations (of the order of about $10 \%$ versus up to $30 \%$, respectively), except perhaps in the North Pacific Ocean. Since diapycnal motions would necessarily be associated with nonconservative effects not accounted for by the present theory and thus able on their own to create diabatic amplitude alterations of the anomalies, it follows that the present calculations in that case only makes sense if the two kind of effects, that is, diabatic versus adiabatic, can be somehow studied separately. Whether this is justified or not requires further work that is beyond the scope of this paper.

While the result that $T^{\prime} / S^{\prime}=\beta_{s / \alpha}$ along the mean streamlines is easy to establish and physically quite intuitive, a more difficult problem concerns the prediction of the respective evolution of $T^{\prime}$ and $S^{\prime}$ along the mean streamlines. From a theoretical viewpoint, this can only be done approximately by constructing a series expansion in terms of the assumed small coupling term between the two prognostic thermodynamic variables. The difficulty that arises stems from the fact that the leading-order prediction depends on the particular choice of prognostic variables considered. Thus, salinity is predicted to be conserved along the mean streamlines when using density and salinity but amplified when using density and spiciness, assuming equatorward propagation from the midlatitudes. More generally, each particular choice of prognostic variables is expected to yield a different leading-order prediction for the respective evolution of $T^{\prime}$ and $S^{\prime}$ along the mean stream- 
lines. The question is therefore which particular choice of prognostic variables provides the "optimal" (in the sense of most accurate) leading-order prediction? One important result of this paper is to provide evidence pointing to the density/spiciness formulation. Indeed, the leading-order prediction of the latter is found to be consistent with that of the second order and hence more accurate density/salinity formulation, in the sense that both predict a dependence upon the ratio $\rho_{\eta} / \rho_{S}$. In contrast, the leading-order density/salinity formulation fails to capture this dependence, which suggests that it is therefore the less accurate of the two formulations.

These results establish that the coupling between density and salinity anomalies can become significant over large distances, but potentially less so between density and spiciness anomalies. Physically, the coupling between the thermodynamic prognostic variables arise from the spatial variations of the haline contraction and thermal expansion coefficients. In this paper, we estimated its value to be $O(10 \mathrm{yr})$. Note, however, that it is not really the particular value of the coupling term that is important to determine whether coupling effects between density and salinity are important over the distance considered, but the fact that the thermodynamic coefficients of seawater vary significantly over such a distance. The $O(1)$ relative spatial variations of $\alpha$ and $\beta_{S}$ in the oceans should also have an impact on the amplitude variations of sea surface height anomalies; so far, the variations of $\alpha$ and $\beta_{S}$ have not been accounted for in the computation of surface wave amplitudes (e.g., Killworth and Blundell 2003b), which may perhaps partly account for some of the discrepancies with observations found in the latter study. Overall, it may be said that the present results complicates seriously the task of deriving amplitude equations that are valid over a whole ocean basin, and future studies should aim at accounting for the effects discussed here.

Further work remains to be done to test the present theoretical predictions. This is arguably not an easy task owing to the paucity of relevant observations. Even if we had sufficient observations, it would arguably remain difficult in view that the present mechanism is not necessarily easily distinguishable from other adiabatic and diabatic mechanisms acting in the ocean interior, like turbulent dissipation, and nonlinear and linear transfer to wave motions. To make progress, carefully designed numerical experiments, such as the ones recently carried out by Schneider (2004), might be useful. This would require some extension of the present theory, since numerical GCM use potential temperature as the conserved quantity, while also accounting for diffusive effects and nonlinearities neglected in this study. Such a study is planned to constitute the second part of this study. In the meantime, assuming that the present leading-order results of the density/spiciness formulation have physical meaning, they have an obvious value in the context of accounting for the observed properties of density-compensated temperature anomalies.

Despite the fact that it neglects many effects such as turbulent diffusion and possible transfer to wave dynamics, our predictions that equatorward propagating density-compensated temperature anomalies should be attenuated are consistent, at least qualitatively, with the properties of seemingly advected temperature anomalies, both observed and numerically simulated in the Pacific (e.g., Schneider et al. 1999), while the situation is less clear in the Atlantic owing to the smaller dimensions of this basin, which reduces the travel time of the anomalies (Lazar et al. 2001). More definitive results, however, must await further study using both a refined theory and high-resolution numerical studies. On the other hand, a potentially important result of this study is that the salinity signature of density-compensated anomalies should reach the equatorial regions relatively unaltered since our theories predict that the latter should either be conserved or amplified during their equatorward journey. This raises the interesting possibility that the thermocline bridge could act as an effective modulator of tropical variability, not through its channeling of temperature anomalies as initially hypothesized by Gu and Philander (1997), but through its channeling of salinity anomalies that will slowly modulate the surface and subsurface salinity balances of the equatorial regions. Empirical studies have found evidence for subducted salinity anomalies originating from midlatitudes reaching the equator (Lukas 2001), but this does not appear to be the only source of salinity variability in the equatorial region (Kessler 1999). The climatic impact of such an effect would be indirect since it would merely act on the stability properties of the mixed layer with various consequences on the vertical extent of heat anomalies, dynamical response to changes in the forcing, and so on. This effect would be potentially important in the regions marked by the existence of a barrier layer for instance, since it is in such regions that the impact of salinity on local heat fluxes and heat content is known to be the most important [e.g., see Vialard and Delecluse (1998a,b) for a modeling study and references].

Acknowledgments. Partial funding for this work from the Innovation Fund of the South African government is gratefully acknowledged.

\section{APPENDIX}

\section{A Derivation of $\rho_{\eta}$ and $\rho_{S}$}

The purpose of this appendix is to relate the coefficients $\rho_{\eta}$ and $\rho_{S}$, which enter the definition of the coupling coefficient $\varpi_{S}$, to the classical haline contraction $\beta_{S}$, thermal expansion $\alpha$, and isothermal compressibility $\gamma$ coefficients. To that end, first note that the state function $\eta=\eta(T, S, p)$, which links the entropy to the 
state variables temperature, salinity, and pressure, can be obtained from the specific Gibbs thermodynamic potential $G=G(S, T, p)$ by $\eta=-G_{T}$ (Feistel and Hagen 1995). [The interested reader may also wish to read Fofonoff (1962), which is the reference on the topic.] Linearizing entropy around mean values of $T, S$, and $p$ yields

$$
\eta^{\prime}=\eta_{T} T^{\prime}+\eta_{S} S^{\prime}+\eta_{p} p^{\prime}
$$

An explicit expressions for the coefficients $\eta_{T}, \eta_{S}$, and $\eta_{p}$ is obtained by taking the relevant partial derivatives of the Gibbs thermodynamic potential as follows:

$$
\eta_{T}=-G_{T T}=\frac{C_{p}}{T}, \quad \eta_{S}=-G_{S T}=-\frac{\partial \mu}{\partial T},
$$

and

$$
\eta_{p}=-G_{T p}=-\frac{\alpha}{\rho},
$$

where $\mu$ is the chemical potential and $C_{p}$ is the heat capacity at constant pressure. From the relation $\rho^{\prime}=$ $\rho_{\eta} \eta^{\prime}+\rho_{S} S^{\prime}+c_{s}^{-2} p^{\prime}$ and (A1), one arrives at

$\rho^{\prime}=\underbrace{\rho_{\eta} \eta_{T} T^{\prime}}_{-\rho \alpha}+\underbrace{\left(\rho_{\eta} \eta_{S}+\rho_{S}\right)}_{\rho \beta_{S}} S^{\prime}+\underbrace{\left(1 / c_{s}^{2}+\rho_{\eta} \eta_{p}\right)}_{\rho \gamma} p^{\prime}$,

where $\alpha, \beta_{S}$, and $\gamma$ are the traditional thermal expansion, haline contraction, and isothermal compressibility coefficients, respectively. By comparing (A3) and (A2), it is possible to obtain an explicit expressions for the following coefficients:

$$
\begin{aligned}
& \rho_{\eta}=-\frac{\alpha T \rho}{C_{p}}=-\rho^{2} \Gamma, \\
& \rho_{S}=\rho \beta_{S}-\frac{\alpha T \rho}{C_{p}} \frac{\partial \mu}{\partial T}=(1-\kappa) \rho \beta_{S}, \quad \text { and } \\
& \rho_{p}=\frac{1}{c_{s}^{2}}=\rho \gamma-\alpha \rho \Gamma=\left(1-\frac{\alpha \Gamma}{\gamma}\right) \rho \gamma,
\end{aligned}
$$

where $\Gamma=\alpha T /\left(\rho C_{p}\right)$ is the adiabatic lapse rate, and $\kappa=$ $\rho \Gamma \mu_{T} / \beta_{S}$, Q.E.D.

\section{REFERENCES}

Boyer, T. P., and S. Levitus, 1997: Objective Analyses of Temperature and Salinity for the World Ocean at $1 / 4^{\circ}$ Grid. NOAA Atlas NESDIS, 11.

Chelton, D. B., and M. G. Schlax, 1996: Global observations of Rossby waves. Science, 232, 234-238.

Cipollini, P., D. Cromwell, P. G. Challenor, and S. Raffaglio, 2001: Rossby waves detected in global ocean color data. Geophys. Res. Lett., 28, 323-326.

Deser, C., M. Alexander, and M. Timlin, 1996: Upper-ocean thermal variability in the North Pacific during 1970-1991. J. Climate, 9, 1840-1855.

Dewar, W. K., 1998: On "too-fast" baroclinic planetary waves in the general circulation. J. Phys. Oceanogr., 28, 1739-1758.

Dickson, R., J. Meincke, S. A. Malmberg, and A. J. Lee, 1988: The "Great Salinity Anomaly" in the northern North Atlantic 1968-1982. Progress in Oceanography, Vol. 20, Pergamon, $103-151$.
Feistel, R., and E. Hagen, 1995: On the GIBBS thermodynamic potential of sea water. Progress in Oceanography, Vol. 36, Pergamon, 249-327.

Flament, P., 2002: A state variable for characterizing water masses and their diffusive stability: spiciness. Progress in Oceanography, Vol. 54, Pergamon, 493-501.

Fofonoff, N. P., 1962: Physical properties of seawater. The Sea, M. N. Hill, Ed., Ocean Engineering Science, Vol. 1, John Wiley and Sons, 3-30.

Fu, L. L., and D. B. Chelton, 2001: Large-scale ocean circulation. Satellite Altimetry and Earth Sciences, L. L. Fu and A. Cazenave, Eds., International Geophysics Series, Vol. 69, Academic Press, 133-169.

Gallego, B., and P. Cessi, 2000: Exchange of heat and momentum between the atmosphere and the ocean: A minimal model of decadal oscillations. Climate Dyn., 16, 479-489.

Gu, D., and S. G. H. Philander, 1997: Interdecadal climate fluctuations that depend on exchanges between the tropics and extratropics. Science, 275, 805-807.

Hill, K. L., I. S. Robinson, and P. Cipollini, 2000: Propagation characteristics of extratropical planetary waves observed in the ATSR global sea surface temperature record. J. Geophys. Res., 105 (C9), 21 927-21945.

Huang, R. X., and J. Pedlosky, 1999: Climate variability inferred from a layered model of the ventilated thermocline. J. Phys. Oceanogr., 29, 779-790.

Kessler, W. S., 1999: Interannual variability of the subsurface high salinity tongue south of the equator at $165^{\circ} \mathrm{W}$. J. Phys. Oceanogr., 29, 2038-2049.

Killworth, P. D., and J. R. Blundell, 2001: Large-scale propagating disturbances: Approximation by vertical normal modes. $J$. Phys. Oceanogr., 31, 2852-2870.

, and — , 2003a: Long extratropical planetary wave propagation in the presence of slowly varying mean flow and bottom topography. Part I: The local problem. J. Phys. Oceanogr., 33, 784-801.

_ , and — , 2003b: Long extratropical planetary wave propagation in the presence of slowly varying mean flow and bottom topography. Part II: Ray propagation and comparison with observations. J. Phys. Oceanogr., 33, 802-821.

- D. Chelton, and R. de Szoeke, 1997: The speed of observed and theoretical long extratropical planetary waves. J. Phys. Oceanogr., 27, 941-962.

Lazar, A., R. Murtugudde, and A. J. Busalacchi, 2001: A model study of temperature anomaly propagation from the subtropics to tropics within the South Atlantic thermocline. Geophys. Res. Lett., 28, 1271-1274.

- T. Inui, P. Malanotte-Rizzoli, A. J. Busalacchi, L. Wang, and R. Murtugudde, 2002: Seasonality of the ventilation of the tropical Atlantic thermocline in an OGCM. J. Geophys. Res., 107, 3104, doi:10.1029/2000JC000667.

Liu, Z., 1999a: Forced planetary wave response in a thermocline gyre. J. Phys. Oceanogr., 29, 1036-1055.

, 1999b: Planetary wave modes in the thermocline: NonDoppler-shift mode, advective mode and Green mode. Quart. J. Roy. Meteor. Soc., 125, 1315-1339.

- and S. Shin, 1999: On thermocline ventilation of active and passive tracers. Geophys. Res. Let., 26, 357-360.

Lukas, R., 2001: Freshening of the upper thermocline in the North Pacific subtropical gyre associated with decadal changes of rainfall. Geophys. Res. Lett., 28, 2485-2488.

Luyten, J., J. Pedlosky, and H. Stommel, 1983: The ventilated thermocline. J. Phys. Oceanogr., 13, 331-371.

Mikolajewicz, U., and E. Maier-Reimer, 1990: Internal secular variability in an ocean general circulation model. Climate Dyn., 4, 145-156.

Polito, P. S., and P. Cornillon, 1997: Long baroclinic Rossby waves detected by TOPEX/POSEIDON. J. Geophys. Res., 102 (C2), 3215-3235.

Quartly, G. D., P. Cipollini, D. Cromwell, and P. G. Challenor, 
2003: Rossby waves: Synergy in action. Philos. Trans. Roy. Soc. London, 361A, 57-63.

Rahmstorf, S., 1995: Bifurcations of the Atlantic thermohaline circulation in response to changes in the hydrological cycle. Nature, 378, 145-149.

Rothstein, L. M., R.-H. Zhang, A. J. Busalacchi, and D. Chen, 1998: A numerical simulation of the mean water pathways in the subtropical and tropical Pacific ocean. J. Phys. Oceanogr., 28, 322-343.

Salmon, R., 1998: Lectures in Geophysical Fluid Dynamics. Oxford University Press, $400 \mathrm{pp}$.

Schneider, N., 2000: A decadal spiciness mode in the tropics. Geophys. Res. Lett., 27, 257-260.

_ 2004: The response of tropical climate to the equatorial emergence of spiciness anomalies. J. Climate, 17, 1083-1095.

_, A. J. Miller, M. A. Alexander, and C. Deser, 1999: Subduction of decadal North Pacific temperature anomalies: Observation and dynamics. J. Phys. Oceanogr., 29, 1056-1070.

Sirven, J., and C. Frankignoul, 2000: Variability of the thermocline due to a sudden change in the Ekman pumping. $J$. Phys. Oceanogr., 30, 1776-1789.

Stommel, H., 1979: Determination of water mass properties of water pumped down from the Ekman layer to the geostrophic flow below. Proc. Natl. Acad. Sci. USA, 76, 3051-3055.

Sturges, W., and B. G. Hong, 1995: Wind forcing of the Atlantic thermocline along $32^{\circ} \mathrm{N}$ at low frequencies. J. Phys. Oceanogr., 25, 1706-1715.

Sutton, R. T., and M. R. Allen, 1997: Decadal predictability of North Atlantic sea surface temperature and climate. Nature, 388, 563-567.

Tailleux, R., and J. C. McWilliams, 2001: Bottom pressure decoupling and the speed of extratropical baroclinic Rossby waves. J. Phys. Oceanogr., 31, 1461-1476.

Vialard, J., and P. Delecluse, 1998a: An OGCM study for the TOGA decade. Part I: Role of salinity in the physics of the western Pacific fresh pool. J. Phys. Oceanogr., 28, 1071-1088. , and - 1998b: An OGCM study for the TOGA decade. Part II: Barrier-layer formation and variability. J. Phys. Oceanogr., 28, 1089-1106.

White, W. B., Y. Chao, and C. K. Tai, 1998: Coupling of biennial oceanic Rossby waves with the overlying atmosphere in the Pacific basin. J. Phys. Oceanogr., 28, 1236-1251.

Williams, R. G., M. Spall, and J. C. Marshall, 1995: Does Stommel's mixed layer "demon" work? J. Phys. Oceanogr., 25, 3089-3102.

Zhang, R. H., and S. Levitus, 1997: Structure and cycle of decadal variability of upper ocean temperature in the North Pacific. $J$. Climate, 10, 710-727.

- T. Kagimoto, and S. E. Zebiak, 2001: Subduction of decadal North Pacific thermal anomalies in an ocean GCM. Geophys. Res. Lett., 28, 2449-2452. 